\title{
Clinical and sebumetric evaluation of topical emulsions in the treatment of acne vulgaris
}

\author{
Barkat Ali Khan, Naveed Akhtar
}

Department of Pharmacy, Faculty of Pharmacy and Alternative Medicine, The Islamia University of Bahawalpur, Bahawalpur, Pakistan Head of Department: Prof. Dr. Naveed Akhtar

Postep Derm Alergol 2014; XXXI, 4: 229-234

DOI: $10.5114 / p d i a .2014 .40934$

\begin{abstract}
Introduction: Numerous plant products described in the scientific literature show distinct activities on the skin, such as moisturizing, antioxidant, sunscreen, anti-acne and depigmentation.

Aim: The main objective of this study was to compare the effectiveness of emulsion formulations containing plant extracts (Hippophae rhamnoides and Cassia fistula) and placebo (without plant extracts) on acne patients.

Material and methods: A single-blind, randomized, placebo-controlled, split-face study was designed. Two groups of 25 patients each (aged 18-37 years) with grade I and grade II acne vulgaris received active formulations on the left side of their cheeks and placebo on the right side of their cheeks twice daily for 12 weeks. Prior to the study, signed consent was obtained from each patient. The anti-bacterial activity of the extracts and formulations was tested in vitro. The skin sebum contents of patients were evaluated by the sebumeter ${ }^{\circledR}$ and subjectively using a clinical evaluation before and after treatment of 12 weeks. One way ANOVA and Kruskal-Wallis tests were used in the statistical analysis.

Results: A significant $(p \leq 0.05)$ decrease in the level of sebum contents was observed in both groups who used formulations (F1 and F2) containing the plant extract. The difference between pre- and post-treatment levels of sebum contents was statistically significant $(p \leq 0.05)$. Formulations containing plant extracts were found effective in the reduction of skin sebum contents (anti-acne effects) sebumetrically as well as clinically when compared to placebo (F3).

Conclusions: Formulations with $5 \%$ plant extracts could be effective, safe, and well-tolerated topical medications for grade I and grade II acne vulgaris.
\end{abstract}

Key words: sebumeter, acne, Cassia fistula, Hippophae rhamnoides.

\section{Introduction}

Acne vulgaris is the most common skin condition that affects adolescents and young adults of 11 to 30 years of age. Acne vulgaris is a multi-factorial disease affecting the pilosebaceous follicles of the dermis. There are 4 pathogenic factors involved in the development of acne: increased sebum production, follicular hyper-keratinization, microbial colonization (Staphylococcus epidermidis and Propionibacterium acnes) and the release of inflammatory mediators into the dermis [1]. Sebum plays the key role in the development of acne. Acne begins just before puberty when the sebaceous glands enlarge and reaches maximum occurrence in early adolescence when sebum outputs reach the peak [2]. Acne vulgaris is characterized by formation of comedones, papules, pus- tules, cysts, nodules and often scars, primarily on the face and neck [3]. The common treatment of acne vulgaris includes oral and topical application of comedolytic agents like benzoyl peroxide, azelaic acid, retinoids and oral/topical antibiotics like tetracycline, erythromycin and clindamycin [4]. These agents have several side effects [5]. In contrast to synthetic agents, natural products are safer and cheap so nowadays physicians prefer the formulations containing plant extracts [6]. Numerous plants are used in traditional medicine worldwide as treatment for acne. Some of these have been subjected to in vitro screening but efficacy of such medicine has rarely been tested in targeted patients [5].

Preliminary phytochemical analysis of Cassia fistula (family: Leguminosae) seeds indicated the presence

Address for correspondence: Barkat Ali Khan, Department of Pharmacy, Faculty of Pharmacy and Alternative Medicine, The Islamia University of Bahawalpur, Bahawalpur 63100, Pakistan, phone: +923339732578, fax: +92629255243, e-mail: barki.gold@gmail.com Received: 24.07.2013, accepted: 24.09.2013. 
of relatively high levels of glycosides, anthraquinones, steroids, terpenoids and phenolic compounds like flavonoids, catechin, epicatechin, Kaempferol, Ellagic acid (EA) and Rhein. In the Indian literature, $C$. fistula has been described to be useful in skin diseases [7-9].

Hippophae rhamnoides has been used in modern science and technology for the last 30 years [10]. Various catechins like (+)-gallocatechin and (-)-epigallocatechin have been isolated from $H$. rhamnoides berries [11]

\section{Aim}

The aim of this study was to investigate the effects of formulations containing a phenolic-rich extract of C. fistula and $H$. rhamnoides in vitro and in vivo for antiacne effects.

\section{Material and methods}

Plant materials and preparation of plant extracts

Cassia fistula pods were collected from the Islamia University of Bahawalpur whereas $\mathrm{H}$. rhamnoides berries were purchased from Pak Sea Buckthorn International Skardu, Pakistan. The plant materials were authenticated by a taxonomist, at the Cholistan Institute of Desert Studies (CIDS), The Islamia University of Bahawalpur and voucher specimens were deposited (voucher \# OB-LF04-11-21 and HR-FT-03-11-23) at the herbarium of the Faculty of Pharmacy, Islamia University of Bahawalpur.

Plant materials were air-dried and then powdered. Each powdered plant was extracted with $70 \%$ methanol solution, and after filtration the filtrates were evaporated under vacuum. These extracts were further used for anti-bacterial activity and emulsion preparation.

\section{Drugs and chemicals}

The test organisms, P. acne (ATCC 6919) and S. epidermidis (ATCC 14990) were obtained from the microbial cul-

Table 1. Composition of formulations (\% w/w)

\begin{tabular}{lcc}
\hline Composition & F1/F2 & F3 \\
\hline Liquid paraffin & 24 & 24 \\
\hline Stearic acid & 5 & 5 \\
\hline Span 20 & 2 & 2 \\
\hline Bees wax & 7 & 7 \\
\hline Cetomacrogol & 5 & 5 \\
\hline Tween 80 & 6 & 6 \\
\hline Plant extract & 5 & Nil \\
\hline Preservative & 1 & 1 \\
\hline D/W & 45 & 50 \\
\hline
\end{tabular}

ture collection section of the Biotechnology Department of the Islamia University of Bahawalpur. Paraffin oil, Stearic acid, Tween80, Span20, Bees wax and Cetomacrogol were purchased from Merck, Germany. Clindamycin was taken from sigma. All reagents used were of analytical grade.

\section{In vitro antibacterial screening of plant extracts}

The anti-bacterial activity of the methanolic plant extracts was tested by the agar well diffusion method described by Schillinger and Lücke [12] with a slight modification. The culture plates were prepared by pouring $20 \mathrm{ml}$ of sterile nutrient agar and $1 \mathrm{ml}$ of inoculums suspension was spread uniformly over the agar medium by using sterile inoculums wire loop. Sterile cork borer was used to make wells of $8 \mathrm{~mm}$ diameter in each plate. These plates were labeled and $100 \mu$ l of plant extracts were poured into wells at a concentration of $150 \mathrm{mg} / \mathrm{ml}$ in aseptic environment using laminar flow hood. Subsequently, the plates were incubated for $24 \mathrm{~h}$ at $37^{\circ} \mathrm{C}$ during which the activity was documented by the appearance of inhibitory zones around the wells. Clindamycin was used as the standard at a concentration of $10 \mu \mathrm{g} / \mathrm{ml}$. Each test was performed in triplicate and the antibacterial activities were expressed as the means of diameters $(\mathrm{mm})$ of the zones of inhibitions surrounding wells containing test materials for comparison with the controls [13].

\section{Preparation of formulations}

Oil-in-water (O/W) type emulsions (F1/F2) containing plant extracts and a placebo (F3) without plant extracts were prepared. The composition of the formulations is given in Table 1. The stability of formulations was evaluated for $\mathrm{pH}$, electrical conductivity, centrifugation, temperature stability tests at various temperatures $\left(8 \pm 0.1^{\circ} \mathrm{C}\right.$, $25 \pm 0.1^{\circ} \mathrm{C}, 40 \pm 0.1^{\circ} \mathrm{C}$ and $40 \pm 0.1^{\circ} \mathrm{C}$ with $75 \%$ relative humidity), rheological characteristics and physical nature, i.e. color, creaming and liquefaction.

\section{In vitro antibacterial screening of formulations}

Vats and Sharma method with a slight modification was applied for in vitro antibacterial screening of formulations [14]. Propionibacterium acne and S. epidermidis were incubated in agar medium for $48 \mathrm{~h}$ and adjusted to yield approximately $1 \times 10^{8} \mathrm{CFU} / \mathrm{ml}$. The solidified agar plates were swabbed with inoculums on the surface. The equidistant wells were cut in the plates with help of $8 \mathrm{~mm}$ borer. In each of these wells the formulation solutions (100 mg of formulation/10 ml of D. water) were placed and the plates were kept at ambient temperature for 30 min to allow pre-diffusion prior to incubation at $37^{\circ} \mathrm{C}$ for $72 \mathrm{~h}$ under anaerobic conditions for $P$. acne and under aerobic conditions for $S$. epidermidis.

The zone of inhibition for each formulation was measured and expressed in $\mathrm{mm}$. Then, the activity index (A.I.) and percent inhibition (P.I.) were calculated for formula- 
tions using the following formula: A.I = mean zone of inhibition of formulation/zone of inhibition of standard, P.I. $=$ A.I $\times 100$.

\section{Study design and volunteers}

In this single-blind, placebo-controlled, split-face mono-centric study, conducted at a referral teaching clinic of dermatology, 50 young adolescents (age range: 18-30 years) with moderate facial acne vulgaris (acne grade I and II) were recruited from September 2011 through December 2011. Written informed consent was taken from the patients after explaining the purpose of the study. They were randomized into two groups with 25 patients each. Group I received F1 and F3 while group II received F2 and F3. Tests were carried out on both the right (applying F3) and left (applying F1 or F2) cheeks of volunteers.

Exclusion criteria were acne secondary to other problems; pregnancy or intention to become pregnant; lactation; another dermatological disease of the face; treatment with oral isotretinoin within the previous 3 months; taking any other acne treatment and known hypersensitivity to the study formulations. The study protocol was approved by the institutional ethics committee of the Faculty of Pharmacy, the Islamia University of Bahawalpur (No. 942/Acad). The study was conducted in accordance with the Declaration of Helsinki and good clinical practices guidelines. Measurements were carried out at baseline (week 01) and on week 02, 04, 06, 08, 10 and 12.

Approximately $500 \mathrm{mg}$ of formulations were applied to the cheeks twice daily (mornings and evenings) for a period of 12 weeks.

\section{Sebumetric analysis}

Bio-metrologically, a sebumeter ${ }^{\circledast}$ (Courage \& Khazaka, Germany) with a noninvasive cassette was used in this study for assessing the skin sebum contents. The measuring head of the cassette represents a $64 \mathrm{~mm}^{2}$ measuring section of an opaque plastic tape which is slightly pushed into the cheeks of volunteers for $30 \mathrm{~s}$ to collect sebum. The resulting change in transparency of the tape was measured and the displayed values correspond to the sebum amount on the face in $\mu g$ of sebum $/ \mathrm{cm}^{2}$.

\section{Clinical evaluation}

A single dermatologist recorded all the clinical observations using assessment scale (Leeds grading scale). Overall change in facial acne compared with the beginning of the study was made on the basis of mild, moderate and severe response.

Photographs of only those volunteers, who allowed openly for reproduction, were taken.

\section{Mathematical analysis}

The percentage changes in skin sebum contents were calculated using the following formula: \%Change $=[(\mathrm{TO}$
- Tw)/Tw] × 100, where: T0 - baseline values, Tw - value obtained on weekly measurement.

\section{Statistical analysis}

The values were analyzed using GraphPad Prism version 5 statistical software. Paired sample $t$-test for variation between the active formulations and placebo was performed. Standard Parametric one-way ANOVA with Dunnett Multiple Comparisons Post Test was used to compare the results between baseline values and the values at different weeks. The level of significance was set at $5 \%$.

\section{Patch Test (Burchard Test)}

The patch test was performed to find out any possible sensitivity of formulations (containing plant extracts) on forearms of all volunteers. An area of $5 \mathrm{~cm} \times 4 \mathrm{~cm}$ was marked on the upper hairless forearms. Placebo (F3) was applied to the right forearm while active formulations (F1/ F2) were applied to the left forearm. The areas were covered with a surgical dressing (Patches) containing formulations. Forty eight hours later, the patches were removed and the forearms were washed with physiological saline. Scores were assigned to the formulations on the basis of presence of itching/irritation using a 4 points scale ranging from 0 to 3; where 3 means severe response, 2 moderate, 1 mild and 0 absence of response. All volunteers were requested to observe the degree of itching/ irritation and allocate a score from the same scale.

\section{Results}

\section{In vitro anti-bacterial activity of plant extracts and formulations}

Table 2 summarizes the results of anti-bacterial activity of plant extracts, where inhibition is expressed as the zone of inhibition in $\mathrm{mm}$. Results indicate that H. rhamnoides had a slight high anti-bacterial activity as compared to C. fistula extracts against both bacteria (P. acne and S. epidermidis). Evaluation of the formulations for anti-bacterial activity confirmed that the active formula-

Table 2. Anti-bacterial activity of $H$. rhamnoides and C. fistula extracts

\begin{tabular}{lcc}
\hline Sample & \multicolumn{2}{c}{$\begin{array}{c}\text { Anti-bacterial activity } \\
\text { (xone of inhibition }[\mathrm{mm}] \text { ) }\end{array}$} \\
\cline { 2 - 3 } & $\begin{array}{c}\text { Propionibacterium } \\
\text { acne }\end{array}$ & $\begin{array}{c}\text { Staphylococcus } \\
\text { epidermidis }\end{array}$ \\
\hline H. rhamnoides & $23.5 \pm 0.64^{\mathrm{a}}$ & $20.5 \pm 1.31^{\mathrm{a}}$ \\
\hline C. fistula & $19.5 \pm 0.92^{\mathrm{b}}$ & $18.2 \pm 2.2^{\mathrm{b}}$ \\
\hline Clindamycin & $19.0 \pm 0.25$ & $18.5 \pm 0.01$ \\
\hline
\end{tabular}

Values were based on triplicates and data presented as mean \pm SD. For the column of each sample, values followed by the same letter are not significantly different at $p<0.05(5 \%)$ 
tions (F1 and F2) having plant extracts at 5\% concentration have the inhibitor activity. Placebo (F3) without the plant extract was used as a control sample, and there was no inhibitory effect observed. In vitro anti-bacterial activity of formulations is presented in Table 3.

\section{Patch test}

There was no itching/irritation in 21 and 18 volunteers for F1 and F2, respectively. No severe response of itching/ irritation was observed in any of the volunteers, mild response occurred in 2 and 3 volunteers while moderate erythema occurred in 2 and 4 volunteers for formulation $\mathrm{F} 1$ and F2, respectively, as shown in Table 4.

\section{Sebumetric analysis}

The percent changes in the skin sebum contents following the 12 weeks' treatment with F1 and F3 are shown in Figure 1, whereas the percent changes in the skin sebum contents following the 12 weeks' treatment with F2 and F3 along with statistical analysis are shown in Figure 2.

In the case of F1 and F3, it was found that F3 has shown a mean initial change in skin sebum contents $(0.45 \%)$ after the $1^{\text {st }}$ week and the effect remained on an slight increasing mode after the $12^{\text {th }}$ week up to $5.97 \%$ compared to the baseline sebum contents of the $25 \mathrm{vol}$ unteers.

On the other hand, an excellent reduction in the sebum contents was observed after treatment with F1, i.e. $-7.32 \%$ mean initial reduction in sebum contents after the $1^{\text {st }}$ week, $-9.1 \%$ reduction after 1 month, and $-21.13 \%$ reduction in sebum contents at the end of the $12^{\text {th }}$ week compared to baseline values. After application of one

Table 3. Percentage of anti-bacterial activity of formulations $(n=3)$

\begin{tabular}{ccc}
\hline Sample & \multicolumn{2}{c}{ Anti-bacterial activity } \\
\cline { 2 - 3 } & $\begin{array}{c}\text { Propionibacterium } \\
\text { acne }\end{array}$ & $\begin{array}{c}\text { Staphylococcus } \\
\text { epidermidis }\end{array}$ \\
\hline F1 & $33.5 \pm 2.3$ & $28.5 \pm 1.08$ \\
\hline F2 & $29.8 \pm 1.02$ & $25.5 \pm 2.5$ \\
\hline F3 & 0 & 0 \\
\hline
\end{tabular}

F1 - Formulation containing $\mathrm{H}$. rhamnoides extract, F2 - formulation containing C. fistula extract, $\mathrm{F} 3$ - placebo

Table 4. Score allocated by volunteers to formulations on the basis of itching/irritation

\begin{tabular}{|c|c|c|c|c|}
\hline \multirow{3}{*}{$\begin{array}{c}\text { Formulation } \\
\text { type }\end{array}$} & \multicolumn{4}{|c|}{ Score } \\
\hline & 0 & 1 & 2 & 3 \\
\hline & \multicolumn{4}{|c|}{ No. of volunteers indicating itching/irritation } \\
\hline F1 & 21 & 2 & 2 & 0 \\
\hline F2 & 18 & 3 & 4 & 0 \\
\hline
\end{tabular}

way analysis of variance, ANOVA (Kruskal-Wallis test) it was observed that there was a statistically significant ( $p \leq 0.05)$ difference between F1 and F3. When Dunn's multiple comparison test was applied, there were non-significant (NS) differences between 12 weeks' time intervals.

In the case of F2 and F3, F3 produced a very minute reduction in skin sebum contents with an initial mean reduction of $-0.033 \%$ after the $1^{\text {st }}$ week and the effect was increased to $5.0 \%$ till the $12^{\text {th }}$ week compared to the baseline skin sebum contents. F2 showed an initial mean reduction of $-1.96 \%$ after one treatment while $-18.0 \%$ mean reduction after 12 weeks' treatment. After applying one way analysis of variance, ANOVA (Kruskal-Wallis test) it was noticed that there was a significant ( $p \leq 0.05)$ difference between F2 and F3. Dunn's multiple compar-

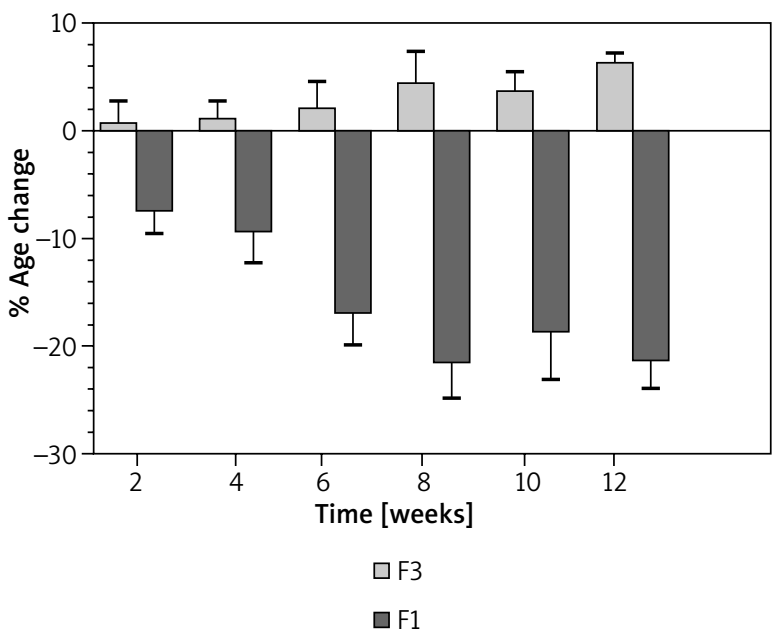

Figure 1. Percentage of changes produced in skin sebum contents after the application of F3 (placebo) and F1 (H. rhamnoides)

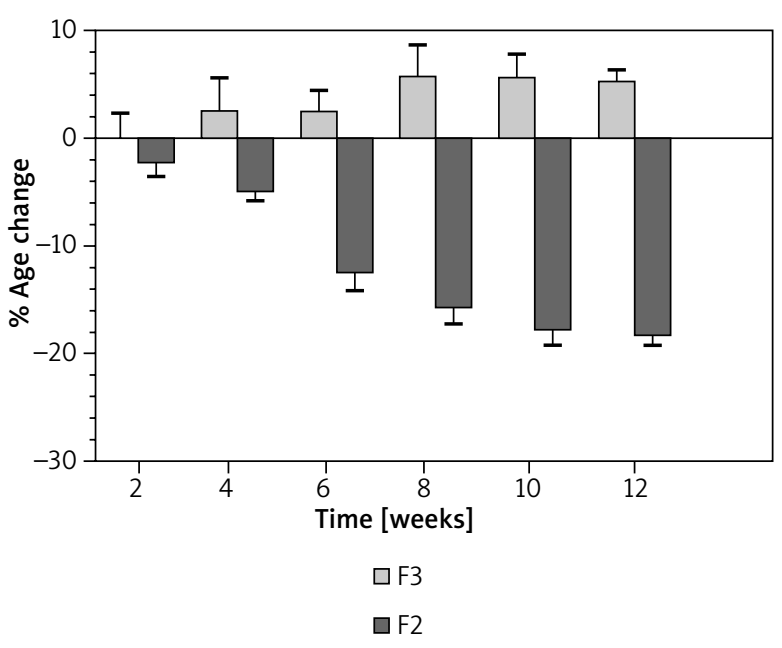

Figure 2. Percentage of changes produced in skin sebum contents after the application of F3 (placebo) and F2 (C. fistula) 
ison test application, there were insignificant $(p>0.05)$ differences between various time intervals except the $1^{\text {st }}$ and $8^{\text {th }}$ week, where significant results were obtained.

\section{Clinical evaluation}

Out of 50 volunteers, 31 (17 for F1 and 14 for F2) cases had grade I acne while 19 (12 for F1 and 07 for F2) cases had grade II acne. In the case of grade I acne, there was an excellent recovery in 9 cases and good results in 17 patients. In the case of grade II acne, there were excellent results in 4 cases and good results in 13 patients. Results are summarized in Table 5. The photograph of a patient is presented in Figure 3.

\section{Discussion}

Acne severity has a connection with the amount of sebum produced. Increased desquamation of follicular keratinocytes and increased cohesiveness of the corneocytes is seen in follicles affected by acne. Increased sebum and desquamated epithelial cells results in follicular obstruction which causes the formation of a micro-comedone and leads to the lesion of acne. Increased sebum also provides a suitable environment for the propagation of $P$. acnes bacteria that settle in the sebum-rich follicles and utilize lipids of sebum as a nutrient source. Lipases released from $P$. acnes break sebum triglycerides into free fatty acids, which are an irritant to the follicular wall and the surrounding dermis after follicular rupture. Propionibacterium acnes bacteria also release pro-inflammatory and chemotactic factors that contribute to the inflammatory response observed in acne $[15,16]$. It is observed that both, the extracts alone and formulations containing extracts showed in vitro anti-bacterial activities. The patch (skin compatibility) test showed that there was no volunteer sensitive to the formulations. Clinically significant ( $p \leq 0.05$ ) results were obtained both for F1 and F2 in the case of grade I and grade II acne.

In this study it has been noted that the F3 (placebo) significantly increased the sebum contents throughout the study period of 12 weeks. In the case of F1 and F2, a regular decline was observed in the sebum contents throughout the study period of 12 weeks.

$5 \alpha$-Reductase converts testosterone into dihydrotestosterone which is more potent and results in the enlargement of sebaceous gland secreting a high amount

Table 5. Clinical evaluation of F1, F2 and F3

\begin{tabular}{|c|c|c|c|c|c|c|c|c|c|c|c|c|}
\hline \multirow[t]{3}{*}{ Grade } & \multirow{3}{*}{$\begin{array}{l}\text { Number } \\
\text { of patients }\end{array}$} & \multirow[t]{3}{*}{ Main features } & \multicolumn{9}{|c|}{ Response } & \multirow[t]{3}{*}{ Value of $p$} \\
\hline & & & \multicolumn{3}{|c|}{ Excellent } & \multicolumn{3}{|c|}{ Good } & \multicolumn{3}{|c|}{ Equivocal } & \\
\hline & & & $\mathrm{F} 1$ & $\mathrm{~F} 2$ & F3 & $\mathrm{F} 1$ & F2 & F3 & $\mathrm{F} 1$ & $\mathrm{~F} 2$ & F3 & \\
\hline 1 & 31 & Papules & 5 & 04 & 00 & 11 & 06 & 00 & 02 & 03 & - & 0.002 \\
\hline \multirow[t]{2}{*}{ II } & 19 & Papules & 2 & 2 & 0 & 8 & 5 & 0 & 1 & 1 & - & 0.047 \\
\hline & & Comedones & 4 & 0 & 0 & 8 & 5 & 0 & 1 & 1 & - & \\
\hline
\end{tabular}
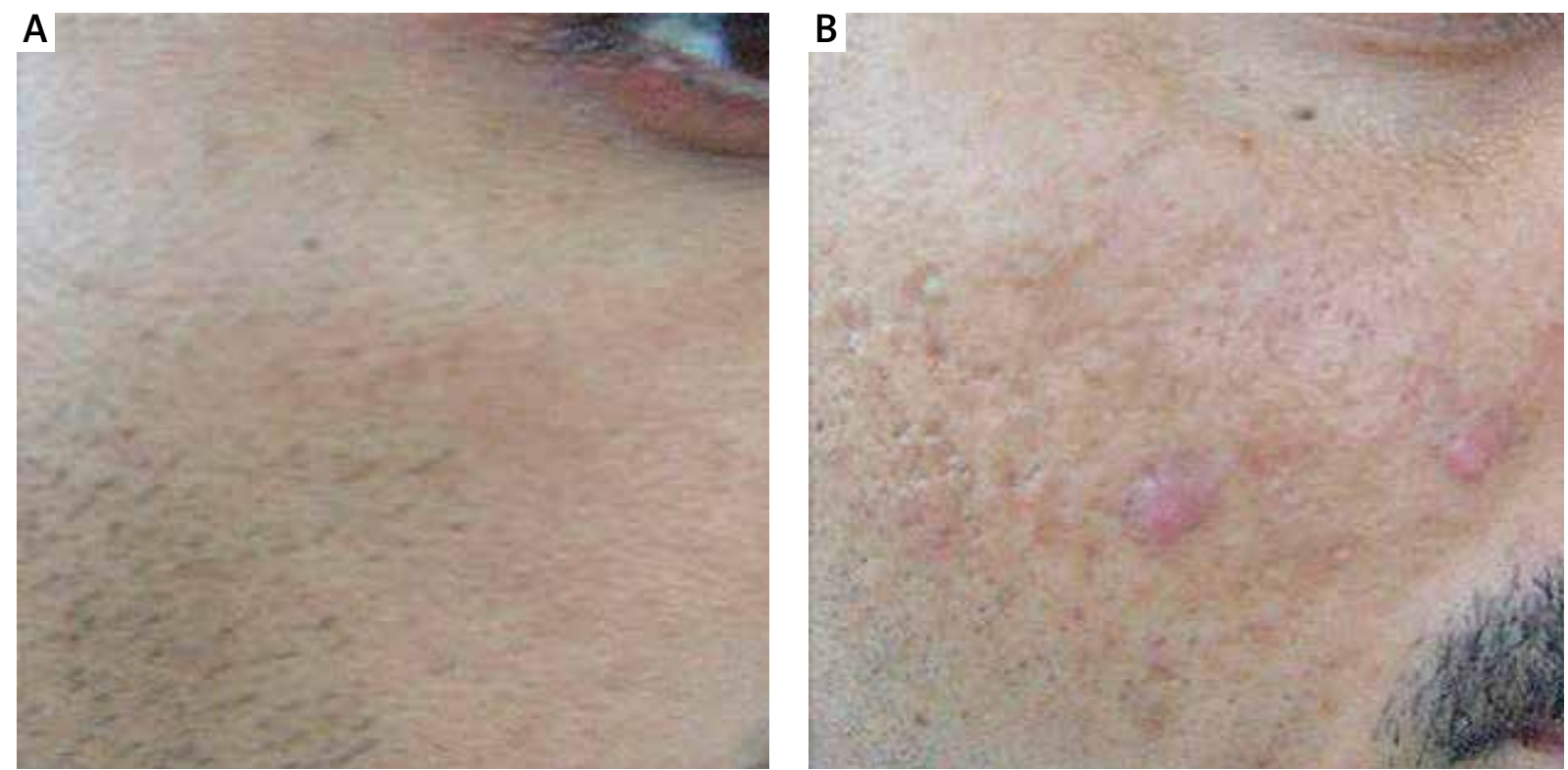

Figure 3. A - Baseline photo of a patient treated with F1. B - Clinical improvement seen after 12 weeks of therapy with F1 
of sebum. It is thought that inhibiting $5 \alpha$-reductase may be effective in lowering the sebum level. Many botanical compounds are considered to inhibit $5 \alpha$-reductase. These include essential fatty acids ( $\gamma$-linolenic acid, $\alpha$-linolenic acid, linoleic, and oleic acids) and phytosterols [17, 18]. Polyphenols in $\mathrm{H}$. rhamnoides and C. fistula include flavonols, catechins, proanthocyanidins and chlorogenic acids while the main fatty acids of $\mathrm{H}$. rhamnoides and $\mathrm{C}$. fistula are palmitoleic acid, palmitic acid, linoleic acid and oleic acid. Due to the presence of these constituents, the formulations reduced sebum contents by possibly inhibiting $5 \alpha$-reductase [19].

\section{Conclusions}

Acne can produce negative psycho-social effects in affected individuals. These include diminished self-esteem, social withdrawal due to discomfiture and depression. Formulations containing plant extracts are believed to be cost effective and free from side effects such as local irritation, scaling, photosensitivity, itching, erythema and skin shedding. The good agreement between the results of in vitro/in vivo analysis and those of clinical evaluation showed that the plant extracts possess efficacy in comparison to placebo in combating acne. The plant extracts, which demonstrated a significant anti-acne activity, were described for the first time to possess this biological property especially in the form of formulation development. Our study shows that formulations containing $H$. rhamnoides and $C$. fistula extracts can be an effective therapy in grade I and II acne vulgaris.

\section{Acknowledgments}

The authors thank to the Higher Education Commission for financial support.

\section{Conflict of interest}

The authors have no competing interests.

\section{References}

1. Diane T. New treatments and therapeutic strategies for acne. Arch Fam Med 2000; 9: 179-87.

2. Abbasi MA, Ayesha K, Aziz UR, et al. Preparation of new formulations of anti-acne creams and their efficacy. Afr J Pharm Pharmacol 2010; 4: 298-303.

3. Lalla JK, Nandedkar SY, Paranjape MH, Talreja NB. Clinical trials of Ayurvedic formulations in the treatment of acne vulgaris. J Ethnopharmacol 2001; 78: 99-102.

4. Clark C. Acne general practice management. The Practioner 1993; 237: 160-4

5. Yadav N, Singh A, Chatterjee A, Belemkar S. Evaluation of efficacy and safety of perfact face gel and perfact face tablets in management of acne. J Clin Exp Dermatol Res 2011; 2: $118-24$.
6. Jain A, Basal E. Inhibition of Propionibacterium acnes-induced mediators of inflammation by Indian herbs. Phytomed 2003; 10: 34-8.

7. Nayan R, Bhalodia, Acharya RN, Shukla VJ. Evaluation of in vitro antioxidant activity of hydroalcoholic seed extratcs of Cassia fistula. Free Rad Antiox 2011; 1: 68-76.

8. Alam MM, Siddiqui MB, Hussian W. Treatment of diabetes through herbal drugs in rural India. Fitother 1990; 61: 240-2.

9. Asolkar LV, Kakkar KK, Chakre OJ. Second supplement to glossary of Indian medicinal plant with active principles. In: Publication and Information Directorate, New Delhi, CSIR 1992; 177-85.

10. Yuzhen Z, Fuheng W. The flavonoids in Seabuckthorn and their medicinal value. Hippophae 1997; 10: 39-41.

11. Rösch D, Krumbein A, Kroh LW. Antioxidant gallocatechins, dimeric and trimeric proanthocyanidins from sea buckthorn (Hippophaë rhamnoides) pomace. Eur Food Res Technol 2004; 219: 605-13.

12. Schillinger $U$, Lücke FK. Antibacterial activity of Lactobacillus sake isolated from meat. Appl Env Microbiol 1989; 55: 1901-6.

13. Khyade MS, Vaikos MN. Pharmacognostical and Phytochemical evaluation of leaf of Jatropha gossipifolia. Intl J Res Ayurved Pharm 2011; 2: 177-81.

14. Vats A, Sharma P. Formulation and evaluation of topical formulation of coriander oil with penetration enhancer. Am J Pharm Technol Res 2012; 2: 452-64.

15. Cunliffe WJ. Acne vulgaris: pathogenesis and treatment. BMJ 1980; 280: 1394-6.

16. Thiboutot DM. An overview of acne and its treatment. Cutis 1996; 57: 8-12

17. Dobrev H. Clinical and instrumental study of efficacy of a new sebum control cream. J Cosmet Dermatol 2007; 6: 113-8.

18. Khan BA, Akhtar N, Mahmood T, et al. Formulation and evaluation of anti sebum secretion effects of Sea Buckthorn W/O emulsion. J Pharm Bioall Sci 2010; 1: 13-7.

19. Rsch D, Bergmann M, Knorr D, Kroh W. Structure-antioxidant efficiency relationships of phenolic compounds and their contribution to the antioxidant activity of Sea Buckthorn juice. J Agri Food Chem 2003; 51: 4233-9. 